\section{OPEN ACCESS}

Edited by:

Zaid A. Abassi,

Technion Israel Institute of

Technology, Israel

Reviewed by:

Michael Haase,

Deutsches Zentrum für

Diabetesforschung (DZD), Germany

Noa Berar Yanay,

Hillel Yaffe Medical Center, Israel

*Correspondence:

Claudius Speer

Claudius.Speer@

med.uni-heidelberg.de

Specialty section:

This article was submitted to

Nephrology,

a section of the journal

Frontiers in Medicine

Received: 06 June 2021

Accepted: 26 July 2021

Published: 17 August 2021

Citation:

Speer C, Morath C, Töllner M,

Buylaert M, Göth D, Nusshag C,

Kälble F, Schaier M, Grenz J,

Kreysing M, Reichel P, Hidmark A,

Ponath G, Schnitzler P, Zeier M,

Süsal C, Klein K and Benning L (2021)

Humoral Responses to Single-Dose

BNT162b2 mRNA Vaccination in

Dialysis Patients Previously Infected

With SARS-CoV-2.

Front. Med. 8:721286.

doi: 10.3389/fmed.2021.721286

\title{
Humoral Responses to Single-Dose BNT162b2 mRNA Vaccination in Dialysis Patients Previously Infected With SARS-CoV-2
}

\begin{abstract}
Claudius Speer ${ }^{1,2 *}$, Christian Morath ${ }^{1}$, Maximilian Töllner ${ }^{1}$, Mirabel Buylaert ${ }^{1}$, Daniel Göth ${ }^{1}$, Christian Nusshag ${ }^{1}$, Florian Kälble ${ }^{1}$, Matthias Schaier ${ }^{1}$, Julia Grenz', Martin Kreysing ${ }^{3}$, Paula Reichel ${ }^{1}$, Asa Hidmark ${ }^{1}$, Gerald Ponath ${ }^{1}$, Paul Schnitzler ${ }^{4}$, Martin Zeier ${ }^{1}$, Caner Süsal ${ }^{5}$, Katrin Klein ${ }^{1}$ and Louise Benning ${ }^{1}$
\end{abstract}

\footnotetext{
${ }^{1}$ Department of Nephrology, University of Heidelberg, Heidelberg, Germany, ${ }^{2}$ Molecular Medicine Partnership Unit Heidelberg, European Molecular Biology Laboratory (EMBL), Heidelberg, Germany, ${ }^{3}$ Department of Gastroenterology and Hepatology, University of Heidelberg, Heidelberg, Germany, ${ }^{4}$ Virology, Department of Infectious Diseases, University of Heidelberg, Heidelberg, Germany, ${ }^{5}$ Department of Transplantation Immunology, Institute of Immunology, University of Heidelberg, Heidelberg, Germany
}

Seroconversion rates following infection and vaccination are lower in dialysis patients compared to healthy controls. There is an urgent need for the characterization of humoral responses and success of a single-dose SARS-CoV-2 vaccination in previously infected dialysis patients. We performed a dual-center cohort study comparing three different groups: 25 unvaccinated hemodialysis patients after PCR-confirmed COVID-19 (Group 1), 43 hemodialysis patients after two-time BNT162b2 vaccination without prior SARS-CoV-2 infection (Group 2), and 13 single-dose vaccinated hemodialysis patients with prior SARS-CoV-2 infection (Group 3). Group 3 consists of seven patients from Group 1 and 6 additional patients with sera only available after single-dose vaccination. Anti-S1 lgG, neutralizing antibodies, and antibodies against various SARS-CoV-2 protein epitopes were measured 3 weeks after the first and 3 weeks after the second vaccination in patients without prior SARS-CoV-2 infection, 6 weeks after the onset of COVID-19 in unvaccinated patients, and 3 weeks after single-dose vaccination in patients with prior SARS-CoV-2 infection, respectively. Unvaccinated patients after COVID-19 showed a significantly higher neutralizing antibody capacity than two-time vaccinated patients without prior COVID-19 [median (IQR) percent inhibition 88.0 (71.5-95.5) vs. 50.7 (26.4-81.0); $P=0.018$ ]. After one single vaccine dose, previously infected individuals generated 15- to 34-fold higher levels of anti-S1 lgG than age- and dialysis vintage-matched unvaccinated patients after infection or two-time vaccinated patients without prior SARS-CoV-2 infection with a median (IQR) index of 274 (151-791) compared to 18 (8-41) and 8 (1-21) (for both $P<0.001)$. With a median (IQR) percent inhibition of 97.6 (97.2-98.9), the neutralizing capacity of SARS-CoV-2 antibodies was significantly higher in single-dose vaccinated patients with prior SARS-CoV-2 infection compared to other groups (for both $P<0.01$ ). Bead-based analysis showed high 
antibody reactivity against various SARS-CoV-2 spike protein epitopes after single-dose vaccination in previously infected patients. In conclusion, single-dose vaccination in previously infected dialysis patients induced a strong and broad antibody reactivity against various SARS-CoV-2 spike protein epitopes with high neutralizing capacity.

Keywords: SARS-CoV-2, COVID-19, hemodialysis, immune response, vaccination

\section{INTRODUCTION}

Patients on maintenance hemodialysis are at great risk for severe courses of coronavirus disease 2019 (COVID-19) caused by severe acute respiratory syndrome coronavirus type 2 (SARS$\mathrm{CoV}-2$ ) (1). An urgent need has been issued to prioritize this vulnerable cohort in international vaccine programs and to determine vaccination response (2).

Recently, we and others demonstrated a lower humoral response to BNT162b2 mRNA vaccine in dialysis patients compared to healthy controls, with particularly low seroconversion rates after the first vaccine dose $(3,4)$. After COVID-19 vaccination and infection, neutralizing antireceptor-binding domain antibodies increase during the first 2 months and decline subsequently over time $(5,6)$. However, little is known about differences in the humoral response of dialysis patients after COVID-19 disease and SARS-CoV-2 vaccination.

Rapid population vaccination coverage is being sought, especially in countries with shortage of vaccine and high COVID19 incidence. Humoral and cellular responses following COVID19 disease may make alternative vaccination strategies necessary for previously infected individuals $(7,8)$. Single-dose rather than double-dose administration might be a reasonable vaccination strategy for individuals recovered from prior infection. First studies showed a strong anti-spike antibody response including neutralizing antibodies in healthy individuals following COVID19 disease after only a single-dose of mRNA vaccine (9-12). However, since sensitivity to the BNT162b2 mRNA vaccine is lower in patients on dialysis with limited seroconversion rates after only one vaccine dose, there is an urgent need to determine the success of single-dose vaccination in previously infected patients. This is one of the first studies providing indepth characterization of humoral responses following singledose vaccination in dialysis patients with confirmed prior SARSCoV-2 infection.

\section{MATERIALS AND METHODS}

\section{Study Design}

In this dual-center observational cohort study, we screened 192 patients on dialysis between March 2020 and April 2021 at the Division of Nephrology of the University Hospital of Heidelberg and at the associated Kidney Center Heidelberg for inclusion (Figure 1). Eligible participants were either hemodialysis patients after PCR-confirmed COVID-19 (Group 1), uninfected hemodialysis patients after two-time mRNA BNT162b2 (BioNTech) vaccination (Group 2), or previously COVID-19 infected individuals who received a single-dose of BNT162b2 $\geq 6$ months after infection (Group 3) (Figure 1). Group 3 consists of seven patients from Group 1 and 6 patients without available sera who are not part of Group 1. We exclusively included patients on hemodialysis.

The anti-S1 IgG index, SARS-CoV-2 specific neutralizing antibodies, and a bead-based differentiation of SARS-CoV-2 target antibodies were measured in 43 BNT162b2 vaccinated dialysis patients without prior SARS-CoV-2 infection 3 weeks after the first and 3 weeks after the second vaccination. In 25 unvaccinated dialysis patients after COVID-19, humoral responses were measured 6 weeks after the onset of infection. The onset of infection was defined as the day with the first symptoms and the diagnosis was made by positive PCR. Vaccination was performed twice in all 43 patients of Group 1 at week 0 and 3. To exclude patients with prior SARS-CoV-2 infection or infection during follow-up, we measured antibodies to the nucleocapsid protein before enrollment and after the first and the second vaccination. SARS-CoV-2 infected dialysis patients who died within the first 6 weeks after disease onset were excluded. In addition, 13 dialysis patients received a single-dose of BNT162b2 vaccine at least 6 months after onset of COVID-19 disease and humoral responses were subsequently measured 3 weeks after single-dose vaccination. To exclude a decisive effect of age and dialysis vintage on humoral responses, we performed a subgroup analysis with age-matched and dialysis vintagematched COVID-19 infected $(N=20)$, fully vaccinated $(N=30)$, and previously infected single-dose vaccinated $(N=13)$ dialysis patients, respectively (Supplementary Tables 1-3). To detect breakthrough infections after vaccination or previous infection, we performed rapid SARS-CoV-2 antigen testing before each individual dialysis session (three times weekly) and PCR testing once a week.

The study (ethics number: S-061/2021) was approved by the ethics committee of the University of Heidelberg on $02 / 15 / 2021$ and conducted in accordance with the Declaration of Helsinki. Written informed consent was obtained from all study participants.

\section{Anti-SARS-CoV-2 IgG Assay}

The IgG response against the $S 1$ protein was measured by the SARS-CoV-2 IgG Assay (Siemens, Eschborn, Germany) and the total antibody response against the nucleocapsid protein by the Elecsys Anti-SARS-CoV-2 assay (Roche, Mannheim, Germany) according to the manufacturer's instructions. A semi-quantitative index of $<1$ was classified as negative, and a value of $\geq 1$ or higher as positive. This cutoff for detection gives a specificity of $99.9 \%$ with a sensitivity of $96.4 \%$ for the Siemens spike assay. 


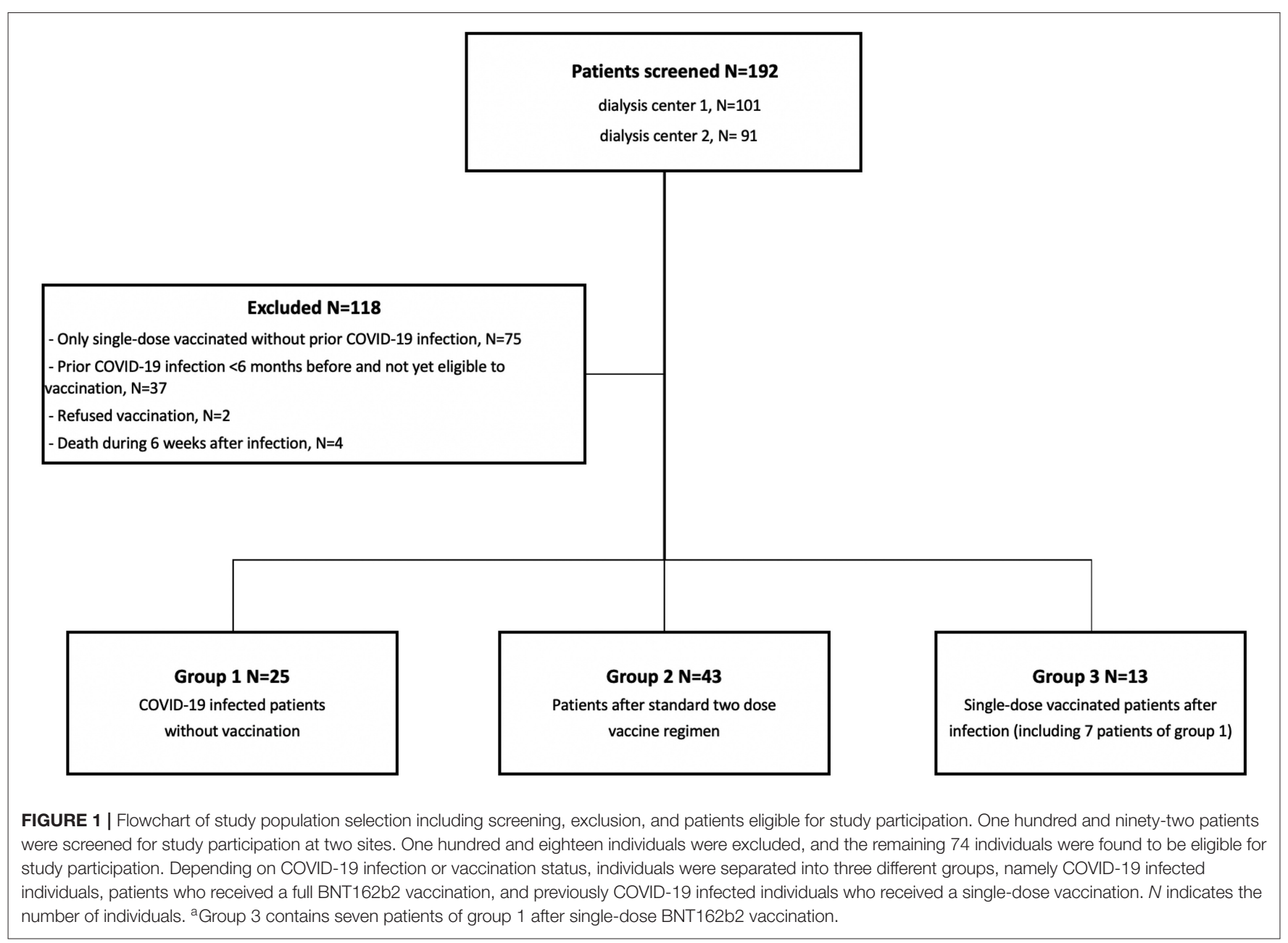

\section{Neutralizing Capacity of SARS-CoV-2 Antibodies}

The binding-inhibition potency of serum samples was detected by a plate-based SARS-CoV-2 surrogate virus neutralizing assay (Medac, Wedel, Germany) $(13,14)$. This assay mimics the virus-host interaction by direct protein-protein interaction using purified receptor-binding domain protein from the viral spike protein and the host cell receptor angiotensin converting enzyme 2 (ACE2). The antibodies in serum samples were incubated with SARS-CoV-2 receptor-binding domain horseradish peroxidase and added to ACE2 coated wells. The reactions were developed using $3,3^{\prime}, 5,5^{\prime}$-tetramethylbenzidine as substrate. Optical density at $450 \mathrm{~nm}$ was measured in each well and the percent (\%) inhibition was calculated as presented in the Supplementary Material. A cut-off for viral neutralization of $\geq 30 \%$ inhibition of receptor-binding domain:ACE- 2 binding was applied according to the manufacturer's instructions, resulting in a specificity of $100 \%$ with a sensitivity of $98 \%$. The SARS-CoV-2 anti-S1 IgG index (Siemens, Eschborn, Germany) correlated with the neutralizing antibody activity measured by the surrogate virus neutralization assay in our cohort (Supplementary Figure 1).

\section{Bead-Based Multiplex Assay for SARS-CoV-2 Antibody Detection}

We determined IgG antibodies against different SARS-CoV-2 target antigens by a multiplex bead-based assay (One Lambda Inc., West Hill, CA) for the Luminex platform (LabScreen COVID Plus), including besides the SARS-CoV-2 nucleocapsid protein, four distinct fragments of the SARS-CoV-2 spike protein, namely the full spike protein, the S1 protein, the receptorbinding domain of the spike protein, and the S2 protein (15). Additionally, we measured S1 fragments from six other coronaviruses, namely HCoV-229E, HCoV-HKU1, HCoV-NL63, HCoV-OC43, MERS-CoV, and SARS-CoV-1. Antibody detection was performed according to the manufacturer's instructions and the mean fluorescence intensity (MFI) was analyzed on a Luminex 200 device (Luminex Corporation, Noord-Brabant, The Netherlands). The MFI cutoff values for each of the 11 proteins are shown in Supplementary Table 4.

\section{Monitoring of Adverse Events}

Adverse events assessment included medical monitoring of local and systemic adverse events for 10 days after the first and the second vaccine dose at each in-house dialysis treatment. Adverse 
TABLE 1 | Baseline characteristics of participating dialysis patients.

\begin{tabular}{|c|c|c|c|c|}
\hline & $\begin{array}{c}\text { Group } 1 \\
\text { (CoVID-19 infected) }\end{array}$ & $\begin{array}{c}\text { Group } 2 \\
\text { (Twice BNT162b2 vaccinated) }\end{array}$ & $\begin{array}{c}\text { Group } 3^{a} \\
\text { (Single-dose BNT162b2 vaccinated after infection) }\end{array}$ & $P$-value \\
\hline Number of patients, $N$ & 25 & 43 & 13 & \\
\hline Age at enrollment (years), median (IQR) & $74(49-82)$ & $82(80-87)$ & 79 (73-84) & $0.002^{b}$ \\
\hline Gender (female), N (\%) & $8(32)$ & $16(37)$ & $2(15)$ & 0.34 \\
\hline BMI, median (IQR) & $26(23-29)$ & $25(22-28)$ & $25(23-27)$ & 0.39 \\
\hline Dialysis vintage (months), median (IQR) & $25(12-64)$ & $44(22-84)$ & $41(19-78)$ & 0.24 \\
\hline \multicolumn{5}{|l|}{ Cause of nephropathy } \\
\hline Diabetes, $N(\%)$ & $5(20)$ & $10(23)$ & $2(15)$ & 0.82 \\
\hline Vascular, N (\%) & $6(24)$ & $8(19)$ & $3(23)$ & 0.85 \\
\hline Polycystic kidney disease, N (\%) & $1(4)$ & $3(7)$ & $0(0)$ & 0.58 \\
\hline Glomerulonephritis, N (\%) & $5(20)$ & $11(26)$ & $4(31)$ & 0.75 \\
\hline Chronic pyelonephritis, N (\%) & $1(4)$ & $1(2)$ & $0(0)$ & 0.75 \\
\hline Other, N (\%) & $7(28)$ & $10(23)$ & $4(31)$ & 0.83 \\
\hline \multicolumn{5}{|l|}{ Comorbidities } \\
\hline Arterial hypertension, $N(\%)$ & $20(80)$ & $43(100)$ & $10(77)$ & $0.006^{b}$ \\
\hline Diabetes, $N(\%)$ & $11(44)$ & $18(42)$ & $5(39)$ & 0.95 \\
\hline Cancer, N (\%) & $6(24)$ & $9(21)$ & $3(23)$ & 0.96 \\
\hline Immunosuppressants, N (\%) & $6(24)$ & $8(19)$ & $3(23)$ & 0.85 \\
\hline Previous transplant, $N(\%)$ & $3(12)$ & $5(12)$ & $1(8)$ & 0.92 \\
\hline Smoker (active and former), N (\%) & $8(32)$ & $15(35)$ & 5 (39) & 0.92 \\
\hline CAD, $N(\%)$ & $16(64)$ & $28(65)$ & $7(71)$ & 0.93 \\
\hline $\mathrm{PAD}, N(\%)$ & $5(20)$ & $15(35)$ & $4(31)$ & 0.43 \\
\hline Chronic lung disease, $N(\%)$ & $7(28)$ & $12(28)$ & $4(31)$ & 0.98 \\
\hline Chronic liver disease, $\underline{\mathrm{N}}(\%)$ & $5(20)$ & $4(9)$ & $2(15)$ & 0.45 \\
\hline
\end{tabular}

$B M I$, body mass index; $C A D$, coronary artery disease; PAD, peripheral artery disease.

${ }^{a}$ Group 3 contains seven patients of Group 1 after single-dose BNT162b2 vaccination and six patients without available sera after infection who are not part of Group 1.

bStatistically significant, results of different groups were compared by applying the Kruskal-Wallis test with Dunn's post-test.

events were assessed using a 12 -item questionnaire inquiring previously mentioned side-effects and the use of pain medication after vaccine reception. The questionnaire is presented in the Supplementary Material. Patients were further screened for infection with COVID-19 three times a week by rapid antigen testing and once a week by PCR testing.

\section{Statistics}

Data are expressed as median and interquartile range (IQR). Results of different groups were compared by applying the Kruskal-Wallis test with Dunn's post-test. Statistical analysis of categorical data was performed using the chi-square $\left(\chi^{2}\right)$ test. Statistical significance was assumed at a $P<0.05$. The statistical analysis was performed using GraphPad Prism version 9.0.0 (GraphPad Software, San Diego CA, USA).

\section{RESULTS}

\section{Baseline Characteristics and Courses of COVID-19 Disease}

Twenty-five patients had a PCR-confirmed COVID-19 disease, 43 patients received the standard two-dose regimen of BNT162b2 mRNA vaccine, and 13 patients received a single-dose BNT162b2 vaccination at least 6 months after the onset of COVID-19 disease
(Figure 1). Baseline characteristics of the different groups are shown in Table 1. We included only patients on hemodialysis in our study. COVID-19 infected patients were significantly younger compared to fully vaccinated or previously infected dialysis patients vaccinated with a single-dose (median age 74 vs. 82 vs. 79 years; $P=0.002$ ). An age- and dialysis vintage-adjusted subgroup analysis was performed and baseline characteristics as well as COVID-19 related clinical characteristics are shown in Supplementary Tables 1, 2, respectively. Antibody responses were determined 6 weeks after the onset of COVID-19 disease, 3 weeks after the first and 3 weeks after the second vaccine dose, or 3 weeks after single-dose vaccination in patients with onset of COVID-19 disease in median (IQR) 6.3 (6.1-6.7) months before.

The clinical courses of individuals infected with SARSCoV-2 are shown in Table 2. According to previously used grading systems for COVID-19 disease severity $(16,17)$, three patients had critical (12\%), three severe (12\%), fourteen moderate (56\%), and five mild (20\%) COVID-19 disease courses (Table 2; Supplementary Table 4). No individual was asymptomatic. Dialysis patients with critical COVID-19 disease courses were more often male and suffered more frequently from diabetes (for both $P<0.05$; Table 2). Four patients died within 6 weeks after onset of COVID-19 disease and were subsequently excluded (Figure 1). 
TABLE 2 | Clinical characteristics and COVID-19 disease courses of dialysis patients with COVID-19 infection.

\begin{tabular}{|c|c|c|c|c|c|c|}
\hline COVID-19 disease severity & Mild ${ }^{b}$ & Moderate & Severe & Critical & All patients & $P$-value \\
\hline Number of patients, $N(\%)$ & $5(20)$ & $14(56)$ & $3(12)$ & $3(12)$ & $25(100)$ & \\
\hline Age at enrollment (years), median (IQR) & $49(43-85)$ & $76(57-82)$ & $75(73-91)$ & $61(46-77)$ & $74(49-82)$ & 0.73 \\
\hline Gender (female), N (\%) & $4(80)$ & $2(14)$ & $2(67)$ & $0(0)$ & $8(32)$ & $0.015^{\mathrm{a}}$ \\
\hline BMI, median (IQR) & 27 (24-29) & $26(23-30)$ & $29(26-30)$ & 27 (23-29) & $26(23-29)$ & 0.84 \\
\hline Dialysis vintage (months), median (IQR) & $36(10-111)$ & $25(8-69)$ & $23(19-72)$ & $34(10-35)$ & $25(12-64)$ & 0.99 \\
\hline \multicolumn{7}{|l|}{ Cause of nephropathy } \\
\hline Diabetes, $N(\%)$ & $0(0)$ & $3(21)$ & $1(33)$ & $1(33)$ & $5(20)$ & 0.69 \\
\hline Vascular, $N(\%)$ & $1(20)$ & $3(21)$ & $1(33)$ & $1(33)$ & $6(24)$ & 0.84 \\
\hline Polycystic kidney disease, N (\%) & $1(20)$ & $0(0)$ & $0(0)$ & $0(0)$ & $1(4)$ & 0.71 \\
\hline Glomerulonephritis, N (\%) & $2(40)$ & $2(14)$ & $0(0)$ & $1(33)$ & $5(20)$ & 0.28 \\
\hline Chronic pyelonephritis, N (\%) & $1(20)$ & $0(0)$ & $0(0)$ & $0(0)$ & $1(4)$ & 0.45 \\
\hline Other, $N(\%)$ & $0(0)$ & $6(43)$ & $1(33)$ & $0(0)$ & $7(28)$ & 0.17 \\
\hline \multicolumn{7}{|l|}{ Comorbidities } \\
\hline Arterial hypertension, $N(\%)$ & $4(80)$ & $11(79)$ & $3(100)$ & $2(67)$ & $20(80)$ & 0.82 \\
\hline Diabetes, $N(\%)$ & $0(0)$ & $6(43)$ & $2(67)$ & $3(100)$ & $11(44)$ & $0.034^{a}$ \\
\hline Cancer, $N(\%)$ & $0(0)$ & $4(29)$ & $1(33)$ & $1(33)$ & $6(24)$ & 0.61 \\
\hline Immunosuppressants, $N(\%)$ & $1(20)$ & $2(14)$ & $1(33)$ & $2(67)$ & $6(24)$ & 0.29 \\
\hline Previous transplant, $N(\%)$ & $1(20)$ & $1(7)$ & $0(0)$ & $1(33)$ & $3(12)$ & 0.47 \\
\hline Smoker (active and former), $N(\%)$ & $1(20)$ & $5(36)$ & $1(33)$ & $1(33)$ & $8(32)$ & 0.86 \\
\hline $\mathrm{CAD}, N(\%)$ & $3(60)$ & $9(64)$ & $1(33)$ & $3(100)$ & $16(64)$ & 0.41 \\
\hline $\mathrm{PAD}, \mathrm{N}(\%)$ & $1(20)$ & $2(14)$ & $0(0)$ & $2(67)$ & $5(20)$ & 0.20 \\
\hline Chronic lung disease, $N(\%)$ & $2(40)$ & $3(21)$ & $1(33)$ & $1(33)$ & $7(28)$ & 0.83 \\
\hline Chronic liver disease, $N(\%)$ & $1(20)$ & $3(21)$ & $0(0)$ & $1(33)$ & $5(20)$ & 0.86 \\
\hline \multicolumn{7}{|l|}{ Initial symptoms } \\
\hline Fever, $N(\%)$ & $3(60)$ & $12(86)$ & $1(33)$ & $3(100)$ & $19(76)$ & 0.31 \\
\hline Cough, $N(\%)$ & $1(20)$ & $7(50)$ & $1(33)$ & $2(67)$ & $11(44)$ & 0.54 \\
\hline Headache, N (\%) & $0(0)$ & $1(7)$ & $0(0)$ & $1(33)$ & $2(8)$ & 0.75 \\
\hline Diarrhea, N (\%) & $1(20)$ & $1(7)$ & $0(0)$ & $1(33)$ & $3(12)$ & 0.81 \\
\hline Fatigue, $N(\%)$ & $3(60)$ & $7(50)$ & $0(0)$ & $2(33)$ & $12(48)$ & 0.38 \\
\hline Dyspnoe, $N(\%)$ & $0(0)$ & $4(29)$ & $3(100)$ & $2(33)$ & $9(36)$ & 0.31 \\
\hline Oxygen supply, N (\%) & $0(0)$ & $4(29)$ & $3(100)$ & $3(100)$ & $10(40)$ & $0.004^{a}$ \\
\hline Non-invasive ventilation, $N(\%)$ & $0(0)$ & $0(0)$ & $3(100)$ & $3(100)$ & $6(24)$ & $0.001^{a}$ \\
\hline Invasive ventilation, $N(\%)$ & $0(0)$ & $0(0)$ & $0(0)$ & $3(100)$ & $3(12)$ & $0.001^{\mathrm{a}}$ \\
\hline Use of vasopressors, $N(\%)$ & $0(0)$ & $0(0)$ & $0(0)$ & $3(100)$ & $3(12)$ & $0.001^{a}$ \\
\hline Days of ICU stay, median (IQR) & 0 & 0 & $4(2-5)$ & $49(43-56)$ & & $0.001^{a}$ \\
\hline Days on ventilation, median (IQR) & 0 & 0 & $2(0-5)$ & $36(23-37)$ & & $0.001^{\mathrm{a}}$ \\
\hline \multicolumn{7}{|l|}{ Immunomodulatory therapy } \\
\hline Dexamethasone, $N(\%)$ & $0(0)$ & $1(7)$ & $2(67)$ & $3(100)$ & $6(24)$ & $0.001^{a}$ \\
\hline Plasma exchange, $N(\%)$ & $0(0)$ & $0(0)$ & $1(33)$ & $2(67)$ & $3(12)$ & $0.006^{a}$ \\
\hline Days of PCR positivity, median (IQR) & $22(17-39)$ & $26(12-35)$ & 34 (18-34) & 43 (29-43) & $29(16-39)$ & 0.32 \\
\hline
\end{tabular}

BMI, body mass index; $C A D$, coronary artery disease; PAD, peripheral artery disease; PCR, polymerase chain reaction; ICU, intensive care unit. a Statistically significant, results of different groups were compared by applying the Kruskal-Wallis test with Dunn's post-test.

${ }^{b}$ Previously used grading systems for COVID-19 disease severity $(16,17)$.

We did not detect symptomatic or asymptomatic breakthrough infections in any of the three different groups during follow-up.

\section{Antibody Responses in Dialysis Patients After COVID-19 Disease or Two Times BNT162b2 mRNA Vaccination}

Dialysis patients after SARS-CoV-2 infection tended to have a higher anti-S1 IgG level as compared to dialysis patients after two times BNT162b2 vaccination (median index 15.8 vs. 6.3; IQR 5.6-37.1 vs. $0.8-14.2 ; P=0.060$; Figure 2A). Whereas, $25 / 25$ (100\%) of SARS-CoV-2 infected dialysis patients had antibody levels above the index cutoff for detection, only 31/43 (72\%) of twice vaccinated patients exceeded this threshold.

We also determined the SARS-CoV-2 neutralizing capacity in both groups. Sera of infected patients showed a significantly higher inhibition than sera of twice vaccinated dialysis patients (median of percent inhibition 88.0 vs. 50.7; IQR 71.5-95.5 vs. 26.4-81.0; $P=0.018$; Figure 2A). An age- and dialysis 


\section{All patients}

A
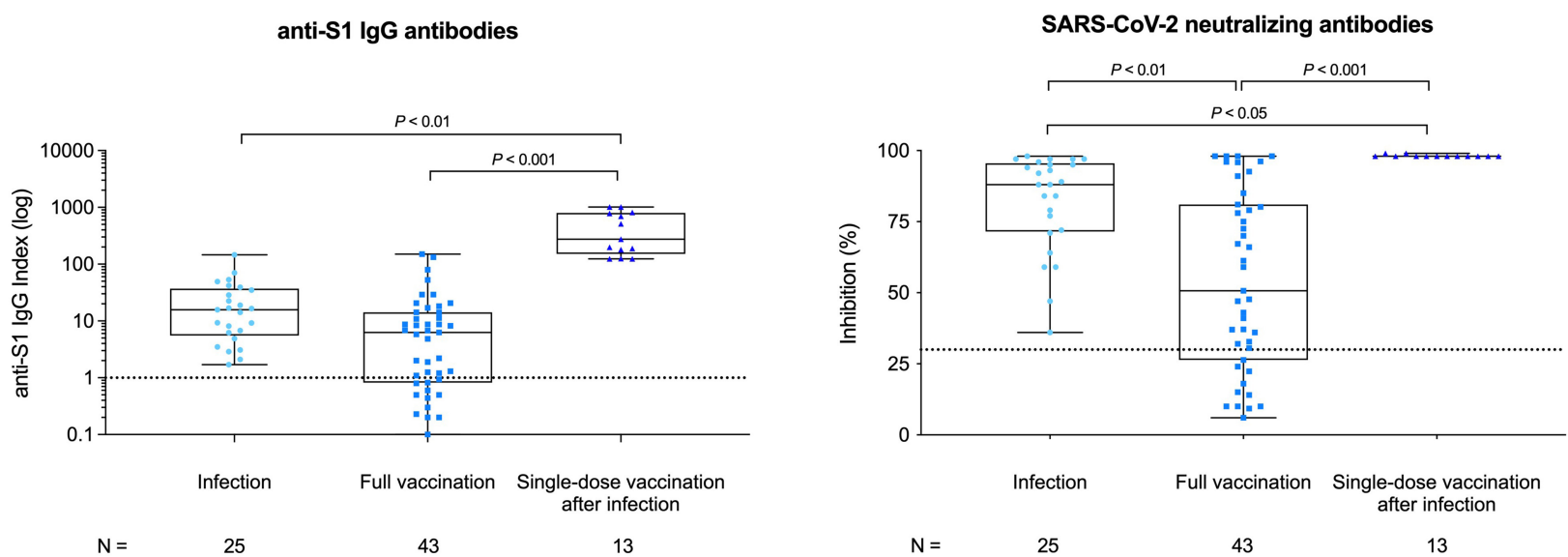

Matched to age and dialysis vintage

B

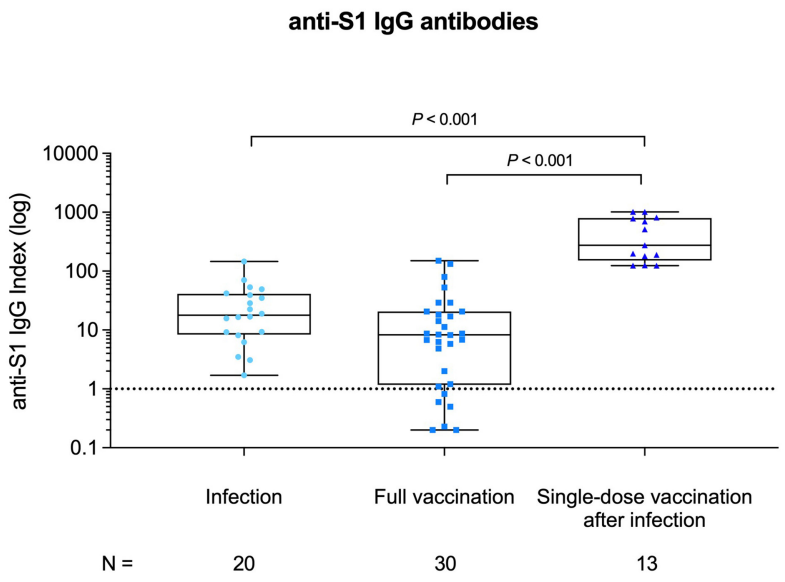

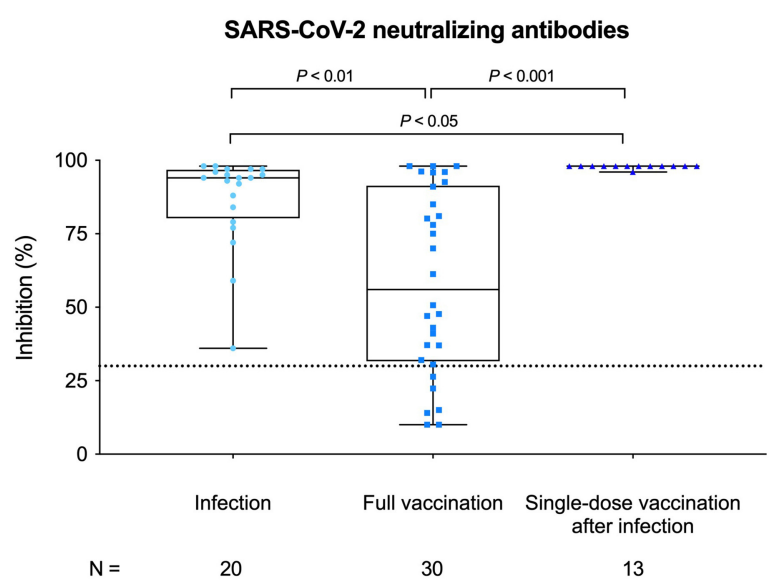

FIGURE 2 | Spike antigen-specific SARS-CoV-2 lgG levels and SARS-CoV-2 neutralizing capacity in hemodialysis patients after COVID-19 infection, complete BNT162b2 vaccination, and in previously infected patients receiving a single dose of BNT162b2. SARS-CoV-2 IgG antibodies and SARS-CoV-2 neutralizing capacity of vaccine-induced antibodies in all (A) and in age- and dialysis vintage-adjusted patients (B) for COVID-19 infected patients, patients receiving complete BNT162b2 vaccination and for previously COVID-19 infected patients receiving a single dose of BNT162b2, respectively. SARS-CoV-2 IgG antibodies are represented logarithmically as an anti-S1 lgG index. The dashed line represents the cutoff for SARS-CoV-2 lgG antibody detection with a semi-quantitative index of $<1$ classified as negative, and a value of $\beta \geq 1$ or higher classified as positive. SARS-CoV-2 neutralizing capacity of BNT162b2 induced antibodies was determined by a virus neutralization test and antibody-mediated inhibition of the SARS-CoV-2 receptor-binding domain:angiotensin-converting enzyme 2 interaction is expressed as a percentage. Binding inhibition $>30 \%$ indicates presence of SARS-CoV-2 neutralizing antibodies above the limit of detection of this test.

vintage-adjusted subgroup analysis revealed similar results for neutralizing antibody capacity $(P=0.047$; Figure 2B; Supplementary Table 3).

Antibodies against the full spike protein, the S1 spike protein, and the receptor-binding domain protein were comparable between both groups with a median (IQR) MFI of 20,862 $(16,750-23,315), 8,908(5,124-13,010)$, and $12,301(7,128-$ $17,355)$ in infected dialysis patients and 21,797 (11,773$23,769), 7,808(2,901-12,556)$, and $12,933(3,929-18,058)$ in fully vaccinated dialysis patients, respectively (Figure 3A). Reactivity of S1 proteins from six other coronaviruses were also comparable between both groups (Supplementary Figure 2). However, infected dialysis patients had higher MFI-values for the spike S2 and the nucleocapsid protein with a median (IQR) of $8,553(6,808-14,041)$ and $13,076(10,452-16,591)$ as compared to fully vaccinated patients with 1,515 (381$4,346)$ and $0(0-139)$ MFI-values $(P=0.007$ and $P<0.001$; Figure 3A). Age- and dialysis vintage-adjusted subgroup analysis confirmed these findings (Figure 3B; Supplementary Table 3). For each individual, the cumulative MFI-value for all SARS-CoV2 proteins tested and the proportional MFI-value as a percentage of each component protein are shown in Figure 4. 

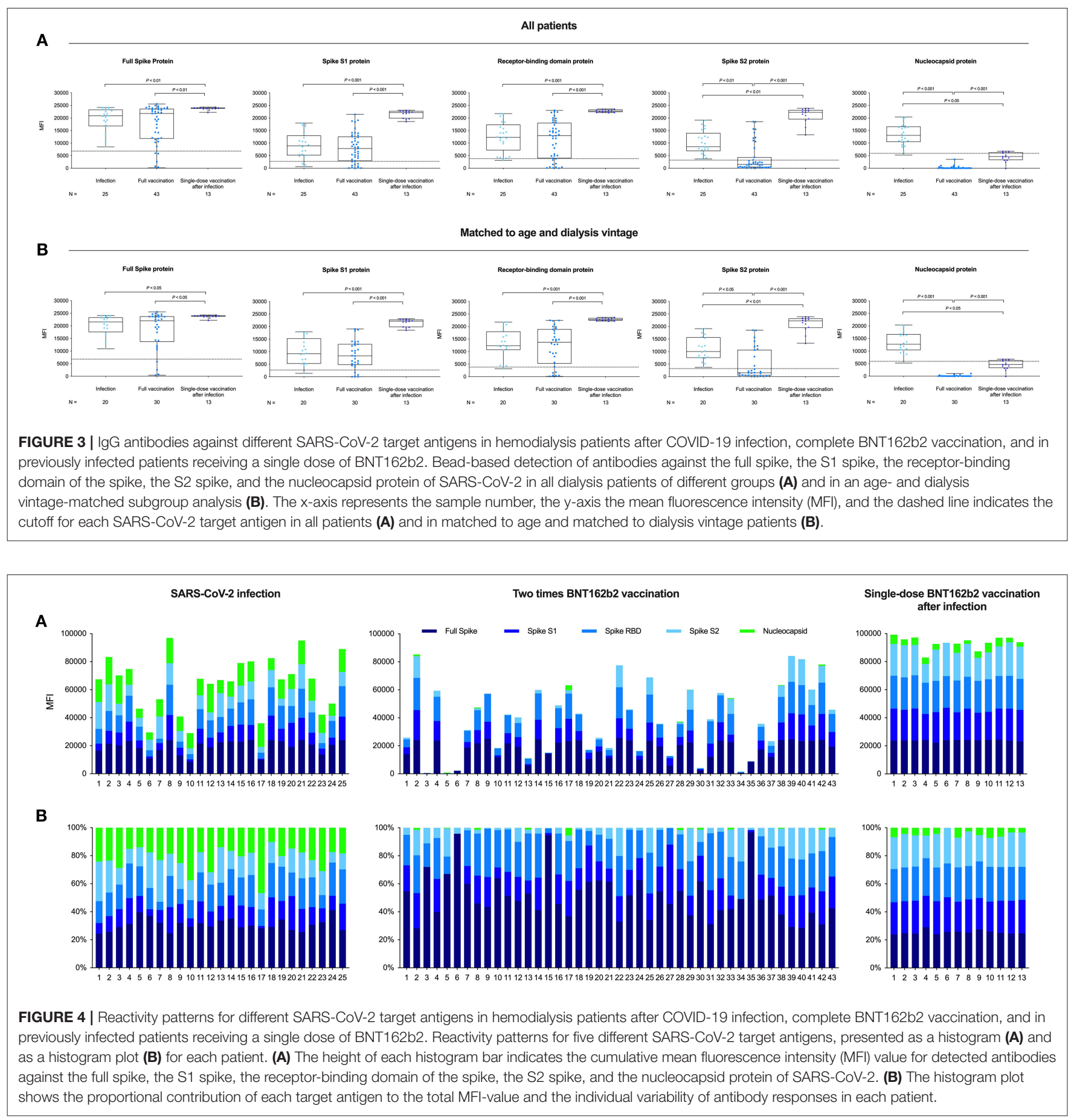

The severity of COVID-19 disease courses did not correlate with results of any of the assays performed (Supplementary Figure 3).

\section{Antibody Response After Single-Dose BNT162b2 mRNA Vaccination in Dialysis Patients With Prior SARS-CoV-2 Infection}

With a median (IQR) of 274 (151-791), dialysis patients with prior infection developed 15- to 34-fold higher anti-S1 IgG levels than age- and dialysis vintage-adjusted patients after infection or after two times vaccination (for both $P<0.001$; Figures 2A,B; Supplementary Table 3).

The inhibition capacity of SARS-CoV-2 antibodies was also significantly higher in previously infected single-dose vaccinated patients compared to patients after infection or patients after two times vaccination, respectively $(P=0.002$ and $P<$ 0.001). Median (IQR) percent inhibition was 97.6 (97.2-98.0) in single-dose vaccinated patients with previous infection as 
compared to 88.0 (71.5-95.5) and 50.7 (26.4-81.0) inhibition in age- and dialysis vintage-adjusted patient after infection or two-times vaccination (Figure 2B; Supplementary Table 3).

Moreover, single-dose vaccinated patients with prior infection showed a strong and broad reactivity against all four different fragments of the SARS-CoV-2 spike protein, whereas reactivity against the nucleocapsid protein was low (Figure 4). All 13 individuals showed MFI-values above the detection cutoff for the full spike, the spike $S 1$, the spike receptor-binding domain, and the spike S2 protein with a median (IQR) MFI of 24,017 $(23,699-$ $24,107), \quad 22,174 \quad(19,849-22,800), \quad 22,736 \quad(22,406-23,337)$, and 22,254 (19,490-23,179), respectively (Figure 3A). Mean fluorescence intensity-values for all four fragments were significantly higher as compared to age- and dialysis vintageadjusted patients after infection or two-times vaccination (Figure 3B; Supplementary Table 3). Reactivity of S1 proteins against the four community coronaviruses and MERS-CoV was comparable between groups, whereas reactivity against the SARS-CoV-1 S1 protein was significantly higher in previously infected dialysis patients vaccinated with a single-dose (for both $P<0.001$; Supplementary Figure 2). Reactivity against the nucleocapsid protein declined in single-dose vaccinated dialysis
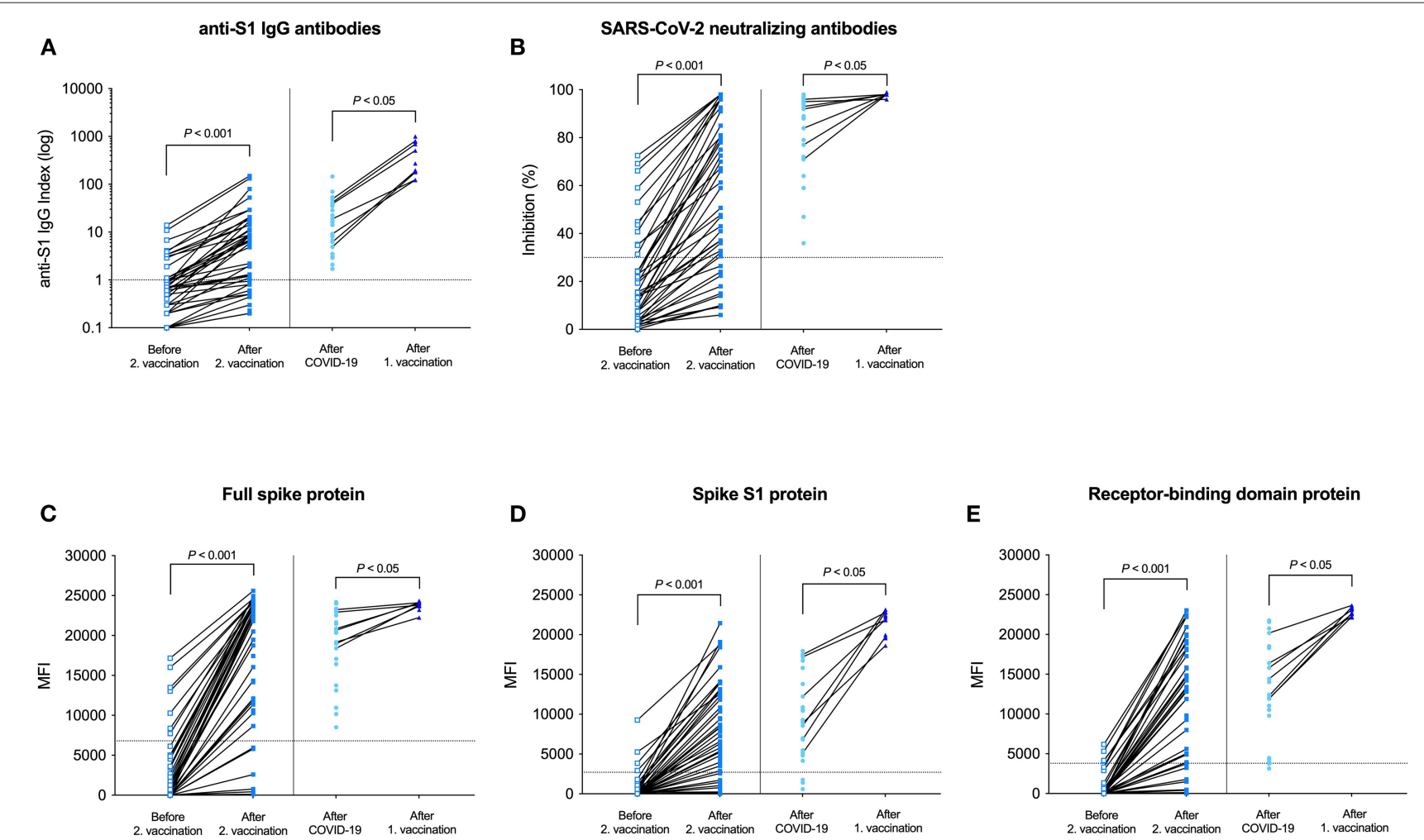

$\mathbf{F}$

Spike S2 protein
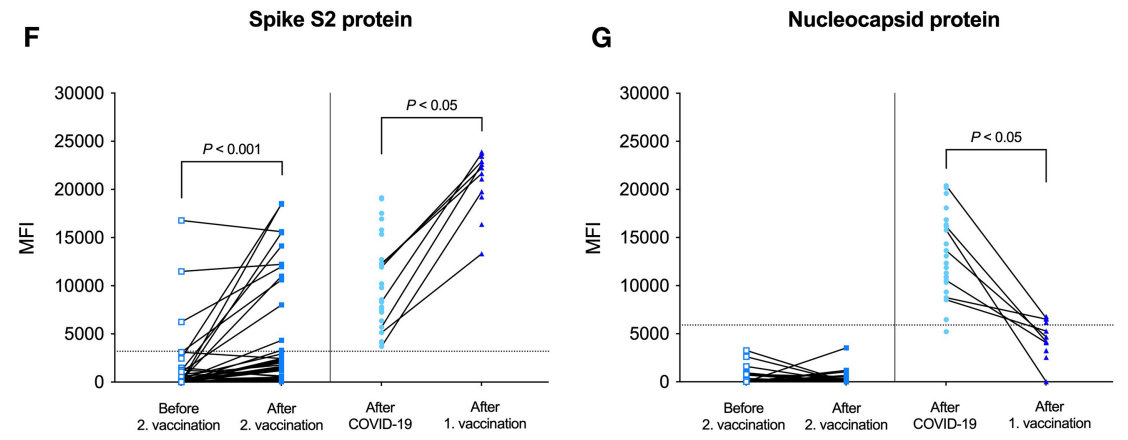

FIGURE 5 | Antibody levels and antibody courses of all measurements from before second vaccination to after second vaccination and from after COVID-19 to after single-dose vaccination in patients with prior infection. SARS-CoV-2 IgG antibodies (A), SARS-CoV-2 neutralizing capacity of vaccine-induced antibodies (B), bead-based detection of antibodies against the full spike (C), the S1 spike (D), the receptor-binding domain of the spike (E), the S2 spike (F), and the nucleocapsid protein of SARS-CoV-2 (G) in hemodialysis patients before second vaccination, after second vaccination, after COVID-19 in unvaccinated patients, and after single-dose vaccination in patients with prior infection. Available individual antibody courses from before second vaccination to after second vaccination and from after COVID-19 to after single-dose vaccination in patients with prior infection are connected by a black line, respectively. 
patients 6 months after SARS-CoV-2 infection (Figure 3A). The median (IQR) MFI of 4,654 (3,251-6,344) was significantly lower as compared to age- and dialysis vintage-adjusted patients 6 weeks after SARS-CoV-2 infection with a median (IQR) MFI of 13,074 (10,452-16,591) $(P<0.05$; Figure 3B).

Figure 5 shows all measurements of humoral responses for all subgroups. Individual antibody courses were determined from before second vaccination to after second vaccination. In addition, antibody courses from after COVID-19 in unvaccinated patients to after single-dose vaccination in patients with prior infection were determined in patients with available sera at both time points (7 out of 13 patients). SARS-CoV-2 antiS1 IgG, neutralizing antibody activity, and reactivity against all four different fragments of the SARS-CoV-2 spike protein significantly increased from before second vaccination to after second vaccination (for all $P<0.001$ ) and from after COVID19 in unvaccinated patients to after single-dose vaccination in patients with prior infection, respectively (for all $P<$ 0.05; Figures 5A-F). In contrast, antibody levels against the nucleocapsid protein significantly decreased over time from after COVID-19 in unvaccinated patients to after singledose vaccination in patients with prior infection $(P<0.05$; Figure 5G).

\section{Local and Systemic Responses After BNT162b2 mRNA Vaccination in Dialysis Patients}

Local responses were low after both the first and second BNT162b2 vaccination in dialysis patients without prior SARSCoV-2 infection (Supplementary Figure 4). Only 2/43 (4.6\%) reported mild-to-moderate pain at the injection site within 2 days after the first injection and 4/43 (9.3\%) after the second injection. Systemic responses were only reported after the second dose of BNT162b2. Systemic events were fatigue 2/43 (4.6\%), headache $2 / 43(4.6 \%)$ and muscle ache $1 / 43$ (2.3\%).

Single-dose vaccination in previously infected dialysis patients was well-tolerated in all patients. Only $3 / 13$ (23.1\%) had pain at the injection site after single-dose vaccination and systemic reactions were not reported (Supplementary Figure 4).

\section{DISCUSSION}

We and others recently showed lower humoral responses to BNT162b2 mRNA vaccine in patients on hemodialysis with particularly low seroconversion rates after the first vaccine dose $(3,4)$. However, most COVID-19 vaccination studies were conducted in healthy individuals and the results have been directly translated to the clinical setting of dialysis patients, although cohort-adapted immunization protocols might be required (18). Since an extremely high incidence of SARS-CoV-2 infection is reported in dialysis patients from countries around the globe, vaccination strategies for previously infected patients urgently needs to be determined (19-22). Recent data suggest that single-dose rather than double-dose BNT162b2 vaccination might be reasonable for healthy individuals recovered from prior infection (12). However, since sensitivity to the first BNT162b2
mRNA vaccine dose is limited in patients on dialysis, success of single-dose vaccination in previously infected patients urgently needs to be confirmed. This is one of the first studies providing in-depth characterization of humoral responses following singledose vaccination in dialysis patients with prior SARS-CoV2 infection.

We investigated the antibody response to single-dose BNT162b2 vaccination in dialysis patients previously infected with SARS-CoV-2 compared with infection-naïve patients receiving standard two-dose vaccination or unvaccinated patients after COVID-19 disease. After symptomatic COVID-19 disease, dialysis patients showed higher SARS-CoV-2 specific neutralizing antibody capacity as compared to two-dose vaccinated patients without prior infection. Most importantly, single-dose vaccinated dialysis patients with prior infection had 15- to 34-fold higher anti-S1 IgG levels than age- and dialysis vintage-adjusted unvaccinated patients after infection or two-time vaccinated patients without prior infection. All 13/13 (100\%) single-dose vaccinated patients with prior infection developed a broad and strong antibody response against all the different SARS-CoV-2 spike protein epitopes, accompanied by an intense neutralizing antibody capacity.

The importance of vaccination in previously infected patients stems from the decreasing SARS-CoV-2 antibody titers over time, which have been described in detail in non-dialysis patients (23). Notably, new emerging variants of concern are posing a challenge, where previously developed antibodies might not suffice to prevent re-infection. Vaccination has shown to add benefit of an increased antibody response against variants of concern such as B.1.351 or P.1 also in previously infected healthy individuals (24). These data highlight the importance of (re)vaccination in immunocompromised patients such as patients on hemodialysis. Because of the low seroconversion rate and the attenuated neutralizing antibody titers after vaccination, this cohort is especially vulnerable for symptomatic infections with upcoming SARS-CoV-2 variants. Recent studies demonstrated that healthy individuals with previous SARS-CoV-2 infection develop strong humoral and cellular responses to a single dose of BNT162b2 $(12,25)$. However, these studies were limited to health care workers only and were small in sample size $(12,25)$. Our own and other studies on humoral responses in infection-naïve patients on hemodialysis have shown low vaccine responses, posing the imminent question whether singledose vaccination in these patients is sufficient $(26,27)$. Attias et al. showed that patients receiving hemodialysis with a previous SARS-CoV-2 infection have high levels of anti-S1 IgG antibodies (28). Since older age was associated with lower seropositivity rate, confirming our own data, we performed an age- and dialysis vintage-adjusted subgroup analysis for every assay (3). We showed for the first time a broad and strong antibody response against all different SARS-CoV-2 spike protein epitopes with a high neutralizing capacity in singledose vaccinated dialysis patients with prior infection. This is even more remarkable because we have only recently shown that a single dose of BNT162b2 mRNA vaccine is unable to exert a protective effect against SARS-CoV-2 in most infectionnaïve patients on hemodialysis. Interestingly, antibodies against 
the nucleocapsid protein declined in single-dose vaccinated patients with prior infection whereas antibodies against the highly immunogenic receptor-binding domain that account for up to $90 \%$ of neutralizing SARS-CoV-2-specific antibodies significantly increased. The lack of vaccine-induced increase in anti-nucleocapsid antibodies in our previously infected patients represents the specificity of BNT162b2 vaccination encoding the full-length SARS-CoV-2 spike protein (29). Given the ongoing shortage of vaccine in most countries around the world, our data suggest that single-dose vaccination in previously infected dialysis patients might be reasonable. However, it remains to be investigated whether enhanced vaccine-induced antibody responses in previously infected dialysis patients will show differential longevity compared to boosted vaccines.

Data from studies published until now indicate that dialysis patients after symptomatic SARS-CoV-2 infection are able to mount a sustained antibody response $(30,31)$. La Milia et al. showed that $15 / 15$ (100\%) symptomatic dialysis patients developed SARS-CoV-2 spike IgG antibodies (30). Titers subsequently declined but seropositivity persisted in all patients after 6 months whereas asymptomatic dialysis patients showed low antibody responses (30). However, little is known about differences in humoral responses between twice vaccinated and SARS-CoV-2 infected dialysis patients. We showed that although anti-S1 IgG antibodies were comparable between infected and twice vaccinated dialysis patients, neutralizing antibody capacity was higher in SARS-CoV-2 infected individuals even after adjusting for age and dialysis vintage. Bead-based analysis of different SARS-CoV-2 specific antibodies revealed comparable antibodies against the receptor-biding domain of the spike protein but a more diverse antibody repertoire with reactivity against the spike S2 protein as well as the nucleocapsid protein after infection. Higher antibody titers have been reported in patients with more severe illness than those with asymptomatic disease in both healthy controls and patients on dialysis (32, 33). We found no association between the severity of COVID19 disease and the level of IgG response or neutralizing antibodies; however, our SARS-CoV-2-infected group did not include asymptomatic individuals. In line with previous studies diabetes and male sex represented risk factors for more severe COVID-19 courses (34).

First studies compared humoral responses of dialysis patients with those of kidney transplant recipients. Rincon-Arevalo et al. showed that most dialysis patients had detectable but significantly lower antibody levels as compared to healthy controls. In contrast, kidney transplant recipients did not develop spikespecific IgG responses, with the exception of one patient who had prior undetected SARS-CoV-2 infection (35). Another study showed seroconversion in $89 \%$ of dialysis patients compared to only $18 \%$ in kidney transplant recipients after two doses of BNT162b2 (36). Because immunization seems to be more efficient in dialysis patients compared to kidney transplant recipients, they concluded that vaccination should be strongly recommended in patients awaiting a kidney transplant (36).

We did not detect breakthrough infections in any of our different groups and data about reinfection after COVID-19 or after SARS-CoV-2 vaccination is reported infrequently until now. Lumley et al. performed a prospective longitudinal cohort study of 12,541 health care workers and found no symptomatic infections and only two PCR-positive results in health care workers with previous anti-spike antibodies (7). They suggest that previous infection, resulting in antibodies to SARS-CoV-2, is associated with protection from reinfection for most people for at least 6 months (7). However, results cannot be directly transferred to dialysis patients and infection- or vaccine-induced protection from asymptomatic or symptomatic (re-)infection needs to be urgently investigated in this cohort.

Although our data conclusively demonstrate a pronounced humoral response in single-dose vaccinated dialysis patients with prior infection, they should be interpreted with caution. First, the extent to which humoral responses contribute to SARS-CoV-2 specific vaccine protection in COVID-19 is still uncertain, and second, there are no universally validated and accepted antibody thresholds that correlate with protection against severe COVID19 courses. Further limitations of our study are the relatively small sample size and the exclusion of infected patients who died during the first 6 weeks after onset of first COVID-19 symptoms. Our preliminary data need to be confirmed by further testing including the cellular immunity, the response against other available vaccines, and most importantly the longevity of induced humoral and cellular responses.

This is one of the first studies to examine in detail the antibody response in previously infected dialysis patients after single-dose BNT162b2 mRNA vaccination. Patients with prior infection developed a broad and strong antibody reactivity against different SARS-CoV-2 spike protein epitopes with a distinct neutralizing capacity after only one vaccine dose. These promising results suggest that single-dose vaccination might also be reasonable in infected dialysis patients as previously shown in healthy controls. Further data on longevity of humoral and cellular vaccine responses are urgently needed to adapt cohortspecific immunization protocols and ultimately protect our most vulnerable cohort-patients on maintenance hemodialysis.

\section{DATA AVAILABILITY STATEMENT}

The raw data supporting the conclusions of this article will be made available by the authors, without undue reservation.

\section{ETHICS STATEMENT}

The studies involving human participants were reviewed and approved by University of Heidelberg. The patients/participants provided their written informed consent to participate in this study.

\section{AUTHOR CONTRIBUTIONS}

CSp, CM, KK, and LB have contributed to planning of the study. CSp, MB, DG, CN, FK, MS, JG, MK, PR, AH, GP, PS, CSü, and LB have contributed to performing the experiments and collecting the data. CSp, CM, MS, AH, GP, PS, MZ, CSü, and LB have contributed to analysis and interpretation of data. CSp, CM, 
GP, PS, MZ, CSü, and LB have contributed to preparation and revision of the manuscript. All authors contributed to the article and approved the submitted version.

\section{FUNDING}

CSp is funded by the Physician Scientist Program of the Heidelberg Faculty of Medicine. LB is funded by the Rahel Goitein-Straus Program of the Heidelberg Faculty of Medicine.

\section{REFERENCES}

1. Chawki S, Buchard A, Sakhi H, Dardim K, Sakhawi KE, Chawki M, et al. Treatment Impact on COVID-19 evolution in hemodialysis patients. Kidney Int. (2020) 98:1053-4. doi: 10.1016/j.kint.2020.07.010

2. Francis A, Baigent C, Ikizler TA, Cockwell P, Jha V. The urgent need to vaccinate dialysis patients against SARS-CoV-2: A call to action. Kidney Int. (2021). 99:791-3. doi: 10.1016/j.kint.2021.02.003

3. Speer C, Göth D, Benning L, Buylaert M, Schaier M, Grenz J, et al. Early humoral responses of hemodialysis patients after COVID-19 vaccination with BNT162b2. Clin J Am Soc Nephrol. (2021) 16:1073-82. doi: 10.2215/cjn.03700321

4. Lesny P, Anderson M, Cloherty G, Stec M, Haase-Fielitz A, Haarhaus M, et al. Immunogenicity of a first dose of mRNA- or vector-based SARS-CoV-2 vaccination in dialysis patients: a multicenter prospective observational pilot study. J Nephrol. (2021) 2021:1-9. doi: 10.1007/s40620-021-01076-0

5. Ma H, Zeng W, He H, Zhao D, Jiang D, Zhou P, et al. Serum IgA, IgM, and IgG responses in COVID-19. Cell Mol Immunol. (2020) 17:773-5. doi: 10.1038/s41423-020-0474-Z

6. Fu Y, Li Y, Guo E, He L, Liu J, Yang B, et al. Dynamics and correlation among viral positivity, seroconversion, and disease severity in COVID-19: a retrospective study. Ann Intern Med. (2021) 174:453-61. doi: $10.7326 / \mathrm{m} 20-3337$

7. Lumley SF, O’Donnell D, Stoesser NE, Matthews PC, Howarth A, Hatch $\mathrm{SB}$, et al. Antibody status and incidence of SARS-CoV-2 infection in health care workers. New Engl J Med. (2020) 384:533-40. doi: 10.1056/nejmoa20 34545

8. Melgaço JG, Azamor T, Bom APDA. Protective immunity after COVID-19 has been questioned: what can we do without SARS-CoV-2-IgG detection? Cell Immunol. (2020) 353:104114. doi: 10.1016/j.cellimm.2020.104114

9. Krammer F, Srivastava K, Alshammary H, Amoako AA, Awawda MH, Beach KF, et al. antibody responses in seropositive persons after a single dose of SARS-CoV-2 mRNA vaccine. New Engl J Med. (2021) 384:1372-4. doi: $10.1056 /$ nejmc2101667

10. Manisty C, Otter AD, Treibel TA, McKnight Á, Altmann DM, Brooks T, et al. Antibody response to first BNT162b2 dose in previously SARS-CoV-2-infected individuals. Lancet. (2021) 397:1057-8. doi: 10.1016/s0140-6736(21)00501-8

11. Wise J. Covid-19: people who have had infection might only need one dose of mRNA vaccine. BMJ. (2021) 372:n308. doi: 10.1136/bmj.n308

12. Saadat S, Tehrani ZR, Logue J, Newman M, Frieman MB, Harris AD, et al. Binding and neutralization antibody titers after a single vaccine dose in health care workers previously infected with SARS-CoV-2. JAMA. (2021) 325:1467-9. doi: 10.1001/jama.2021.3341

13. Tan CW, Chia WN, Qin X, Liu P, Chen MI-C, Tiu C, et al. A SARS-CoV2 surrogate virus neutralization test based on antibody-mediated blockage of ACE2-spike protein-protein interaction. Nat Biotechnol. (2020) 38:1073-8. doi: 10.1038/s41587-020-0631-z

14. Wacharapluesadee S, Tan CW, Maneeorn P, Duengkae P, Zhu F, Joyjinda $\mathrm{Y}$, et al. Evidence for SARS-CoV-2 related coronaviruses circulating in bats and pangolins in Southeast Asia. Nat Commun. (2021) 12:972. doi: 10.1038/s41467-021-21240-1

15. Bray RA, Lee J-H, Brescia P, Kumar D, Nong T, Shih R, et al. Development and validation of a multiplex, bead-based assay to detect antibodies

\section{ACKNOWLEDGMENTS}

We thank Jochen Reiser for critical reading of the manuscript.

\section{SUPPLEMENTARY MATERIAL}

The Supplementary Material for this article can be found online at: https://www.frontiersin.org/articles/10.3389/fmed. 2021.721286/full\#supplementary-material

directed against SARS-CoV-2 proteins. Transplantation. (2020) 105:79-89. doi: $10.1097 /$ tp. 0000000000003524

16. Gandhi RT, Lynch JB, del Rio C. Mild or moderate covid-19. New Engl J Med. (2020) 383:1757-66. doi: 10.1056/nejmcp2009249

17. Madhi SA, Baillie V, Cutland CL, Voysey M, Koen AL, Fairlie L, et al. Efficacy of the ChAdOx1 nCoV-19 Covid-19 vaccine against the B.1.351 variant. New Engl J Med. (2021) 384:1885-98. doi: 10.1056/nejmoa2102214

18. Glenn DA, Hegde A, Kotzen E, Walter EB, Kshirsagar AV, Falk R, et al. Systematic review of safety and efficacy of COVID-19 vaccines in patients with kidney disease. Kidney Int Reports. (2021) 6:1407-10. doi: 10.1016/j.ekir.2021.02.011

19. Hsu CM, Weiner DE, Aweh G, Miskulin DC, Manley HJ, Stewart C, et al. COVID-19 Infection among US dialysis patients: risk factors and outcomes from a national dialysis provider. Am J Kidney Dis. (2021) 77:748.e1-56.e1. doi: 10.1053/j.ajkd.2021.01.003

20. Couchoud C, Bayer F, Ayav C, Béchade C, Brunet P, Chantrel F, et al. Low incidence of SARS-CoV-2, risk factors of mortality and the course of illness in the French national cohort of dialysis patients. Kidney Int. (2020) 98:1519-29. doi: 10.1016/j.kint.2020.07.042

21. Corbett RW, Blakey S, Nitsch D, Loucaidou M, McLean A, Duncan N, et al. Epidemiology of COVID-19 in an urban dialysis center. J Am Soc Nephrol. (2020) 31:1815-23. doi: 10.1681/asn.2020040534

22. Khatri M, Islam S, Dutka P, Carson J, Drakakis J, Imbriano L, et al. COVID19 antibodies and outcomes among outpatient maintenance hemodialysis patients. Kidney360. (2020) 2:263-9. doi: 10.34067/kid.0006292020

23. Dan JM, Mateus J, Kato Y, Hastie KM, Yu ED, Faliti CE, et al. Immunological memory to SARS-CoV-2 assessed for up to 8 months after infection. Science. (2021) 371:eabf4063. doi: 10.1126/science.abf4063

24. Lustig Y, Nemet I, Kliker L, Zuckerman N, Yishai R, Alroy-Preis S, et al. Neutralizing response against variants after SARS-CoV-2 infection and one dose of BNT162b2. New Engl J Med. (2021) 84:2453-4. doi: $10.1056 /$ nejmc2104036

25. Prendecki M, Clarke C, Brown J, Cox A, Gleeson S, Guckian M, et al. Effect of previous SARS-CoV-2 infection on humoral and T-cell responses to single-dose BNT162b2 vaccine. Lancet. (2021) 397:1178-81. doi: 10.1016/s0140-6736(21)00502-x

26. Grupper A, Sharon N, Finn T, Cohen R, Israel M, Agbaria A, et al. Humoral response to the Pfizer BNT162b2 vaccine in patients undergoing maintenance hemodialysis. Clin J Am Soc Nephrol. (2021) 2021:CJN.03500321. doi: 10.2215/cjn.03500321

27. Torreggiani M, Blanchi S, Fois A, Fessi H, Piccoli GB. Neutralizing SARSCoV-2 antibody response in dialysis patients after the first dose of the BNT162b2 mRNA Covid-19 vaccine. The war is far from being won. Kidney Int. (2021) 99:1494-6. doi: 10.1016/j.kint.2021.04.010

28. Attias P, Sakhi H, Rieu P, Soorkia A, Assayag D, Bouhroum S, et al. Antibody response to BNT162b2 vaccine in maintenance hemodialysis patients. Kidney Int. (2021) 99:1490-2. doi: 10.1016/j.kint.2021.04.009

29. Polack FP, Thomas SJ, Kitchin N, Absalon J, Gurtman A, Lockhart S, et al. Safety and efficacy of the BNT162b2 mRNA covid-19 vaccine. New Engl J Med. (2020) 383:2603-15. doi: 10.1056/nejmoa2034577

30. Milia VL, Tonolo S, Luzzaro F, Bonato C, Cavalli A, Foglieni B, et al. The humoral immune response to SARS-CoV-2 mounts and is durable in symptomatic hemodialysis patients. Nephrol Dial Transpl. (2021) 36:1132-34. doi: 10.1093/ndt/gfab047 
31. Vriese ASD, Reynders M. IgG antibody response to SARS-CoV-2 infection and viral RNA persistence in patients on maintenance hemodialysis. Am J Kidney Dis. (2020) 76:440-1. doi: 10.1053/j.ajkd.2020.05.009

32. Röltgen K, Powell AE, Wirz OF, Stevens BA, Hogan CA, Najeeb J, et al. Defining the features and duration of antibody responses to SARS-CoV-2 infection associated with disease severity and outcome. Sci Immunol. (2020) 5:eabe0240. doi: 10.1126/sciimmunol.abe0240

33. Sakhi H, Dahmane D, Attias P, Kofman T, Bouvier M, Lapidus N, Fourati $\mathrm{S}$, et al. Kinetics of anti-SARS-CoV-2 IgG antibodies in hemodialysis patients six months after infection. J Am Soc Nephrol. (2021) 32:1033-6. doi: 10.1681/asn.2020111618

34. Richardson S, Hirsch JS, Narasimhan M, Crawford JM, McGinn T, Davidson KW, et al. Presenting characteristics, comorbidities, and outcomes among 5700 patients hospitalized with COVID-19 in the New York City area. JAMA. (2020) 323:2052-9. doi: 10.1001/jama.20 20.6775

35. Rincon-Arevalo H, Choi M, Stefanski AL, Halleck F, Weber U, Szelinski F, et al. Impaired humoral immunity to SARS-CoV-2 BNT162b2 vaccine in kidney transplant recipients and dialysis patients. Sci Immunol. (2021) 6:eabj1031. doi: 10.1126/sciimmunol.abj1031

36. Bertrand D, Hamzaoui M, Lemée V, Lamulle J, Hanoy M, Laurent C, et al. Antibody and T cell response to SARS-CoV-2 messenger RNA BNT162b2 vaccine in kidney transplant recipients and hemodialysis patients. J Am Soc Nephrol. (2021) 2021:ASN.2021040480. doi: 10.1681/asn.2021040480

Conflict of Interest: The authors declare that the research was conducted in the absence of any commercial or financial relationships that could be construed as a potential conflict of interest.

Publisher's Note: All claims expressed in this article are solely those of the authors and do not necessarily represent those of their affiliated organizations, or those of the publisher, the editors and the reviewers. Any product that may be evaluated in this article, or claim that may be made by its manufacturer, is not guaranteed or endorsed by the publisher.

Copyright (C) 2021 Speer, Morath, Töllner, Buylaert, Göth, Nusshag, Kälble, Schaier, Grenz, Kreysing, Reichel, Hidmark, Ponath, Schnitzler, Zeier, Süsal, Klein and Benning. This is an open-access article distributed under the terms of the Creative Commons Attribution License (CC BY). The use, distribution or reproduction in other forums is permitted, provided the original author(s) and the copyright owner(s) are credited and that the original publication in this journal is cited, in accordance with accepted academic practice. No use, distribution or reproduction is permitted which does not comply with these terms. 